\title{
Differential remodeling of a T-cell transcriptome following CD8- versus CD3-induced signaling
}

\author{
S Hussain I Abidi' ${ }^{1,2}$, Tao Dong ${ }^{1}$, Mai T Vuong ${ }^{1}$, Vattipally B Sreenu ${ }^{1}$, Sarah L Rowland-Jones ${ }^{1}$, \\ Edward J Evans ${ }^{1}$, Simon J Davis ${ }^{1}$ \\ ${ }^{I}$ Nuffield Department of Clinical Medicine and MRC Human Immunology Unit, Weatherall Institute of Molecular Medicine, The \\ University of Oxford, John Radcliffe Hospital, Headington, Oxford OX3 9DS, UK; '² Present address: Department of Cell Biology, \\ Harvard Medical School, 240 Longwood Avenue, Boston, MA 02115, USA
}

CD8 engagement with class I major histocompatibility antigens greatly enhances T-cell activation, but it is not clear how this is achieved. We address the question of whether or not the antibody-mediated ligation of CD8 alone induces transcriptional remodeling in a T-cell clone, using serial analysis of gene expression. Even though it fails to induce overt phenotypic changes, we find that CD8 ligation profoundly alters transcription in the T-cell clone, at a scale comparable to that induced by antibody-mediated ligation of CD3. The character of the resulting changes is distinct, however, with the net effect of CD8 ligation being substantially inhibitory. We speculate that ligating CD8 induces weak, T-cell receptor (TCR)-mediated inhibitory signals reminiscent of the effects of TCR antagonists. Our results imply that CD8 ligation alone is incapable of activating the T-cell clone because it fails to fully induce NFAT-dependent transcription.

Keywords: receptor triggering, signaling, CD8, coreceptor, SAGE, expression analysis

Cell Research (2008) 18:641-648. doi: 10.1038/cr.2008.56; published online 13 May 2008

\section{Introduction}

Understanding how different types of cell surface receptors convey, to the interior of a cell, the information that a binding event has taken place at its surface remains among the most important problems in cell biology. Among the set of receptors involved in activating $\mathrm{T}$ cells, the co-receptors, i.e. $\mathrm{CD} 8$ and $\mathrm{CD} 4$, are perhaps the most enigmatic. The importance of CD8 in cytotoxic T-cell activation is emphasized by the finding that CD8 enhances the antigen sensitivity of these cells by one million-fold or more [1]. Somewhat paradoxically, however, the affinity of the interaction of CD8 with its ligand on antigen-presenting cells, i.e. an MHC class I molecule, is among the lowest that have been described for protein interactions at the cell surface $\left(K_{\mathrm{d}} \sim 200 \mu \mathrm{M}[2]\right)$. The affinity is so low, in fact, that it is not even clear whether it is capable of mediating the independent binding of $\mathrm{CD} 8$ to $\mathrm{MHC}$ proteins at physiological

Correspondence: Edward J Evans ${ }^{\mathrm{a}}$, Simon J Davis ${ }^{\mathrm{b}}$

Tel: +44-1865-221336

aE-mail: edward.evans@ndm.ox.ac.uk

bE-mail: simon.davis@ndm.ox.ac.uk

Received 28 August 2007; revised 13 December 2007; accepted 18 January 2008; published online 13 May 2008 expression levels [3]. Exactly how CD8 contributes to T-cell activation, therefore, constitutes something of a mystery. The observation that $\mathrm{CD} 8 \alpha$ binds with relatively high affinity $(10 \mu \mathrm{M})$ to the thymus leukemia antigen (TL), a non-classical MHC class I molecule [4,5], implies that CD8 might have intrinsic signaling activity. For some T-cell surface receptors, e.g. CD2 [6] and CD28 (referred to in [7]), whose ligation induces cellular responses, signaling is known to depend on the presence of the T-cell receptor (TCR) and its associated signaling apparatus. This suggests that these receptors somehow interact with the TCR and/or promote signaling through TCR-associated intracellular pathways, even in the absence of TCR ligands. We have considered the question of whether or not ligating CD8 alone is capable of inducing any transcriptional remodeling in T cells and, if so, whether these changes differ from those induced by ligation of the TCR complex.

\section{Results and Discussion}

We examined the signaling response in $\mathrm{CD} 8^{+}$clone 32 T-cells, which have been shown to recognize the human immunodeficiency virus-1 pol A peptide ETAYFILKL in the context of MHC class I-A6802 [8]. Clone 32 was chosen because the resting transcriptome of these cells has already 
been characterized at considerable depth and found to be similar to that of a sorted ex-vivo population of $\mathrm{CD} 8^{+} \mathrm{T}$-cells [9]. Cross-linking of CD8 with the anti-CD8 antibody MF8 failed to activate clone $32 \mathrm{~T}$-cells, as judged by the general absence of large changes in the cell surface expression of a suite of cell surface markers, including CD69 and CD25 (Supplementary information, Figure S1). This suggests that the cross-linking of $\mathrm{CD} 8$ induces very weak or no signaling, or that it induces signals that are inhibitory.

To distinguish between these possibilities, we examined the transcriptome of anti-CD8 antibody-treated clone $32 \mathrm{~T}$ cells, and compared it with that of clone $32 \mathrm{~T}$-cells activated via the TCR, using the anti-CD3 antibody, OKT3, as a surrogate TCR ligand. Transcriptome analyses were based on data obtained using the serial analysis of gene expression (SAGE) method, which generates short, transcript-specific tags that can be sequenced in a high-throughput manner, allowing robust quantitative analyses of the transcriptional activities of cells and tissues [10]. Resting, anti-CD8 and anti-CD3 antibody-treated clone 32-derived SAGE libraries were sequenced to similar depths ( $\sim 70000$ tags), thus enabling unbiased comparisons of the three libraries.

Using a confidence interval of $99 \%$, representations of the data as log scatter plots revealed that large changes in gene expression accompany receptor ligation by each antibody (Figure 1A and 1B). In both cases, 400-500 SAGE tags increase in abundance as a proportion of the total set of tags following antibody treatment and even more tags decrease in abundance (Figure 1A and 1B). Similar numbers of tags are significantly over-represented in the resting clone $32 \mathrm{~T}$ cell-derived library versus libraries generated from distinct leukocyte lineages (e.g. a CD4 ${ }^{+} \mathrm{T}$-cell; 561 transcripts) or different tissues (e.g. cerebellum; 631 transcripts) [9], emphasizing the scale of the remodeling that follows signaling by cell surface proteins. The number of tags (and therefore transcripts) affected was only slightly higher (by $20 \%$ ) for anti-CD3 treatment than for anti-CD8 treatment, indicating that the breadth of the transcriptional response to signaling by CD8 is comparable in overall scale to that induced by signaling via the TCR. Somewhat unexpectedly, in both cases, substantially more transcripts (30-50\%) are down-regulated than are up-regulated, implying that a large fraction of the activation response involves gene suppression. It should be noted that apparent up- or down-regulation of a given transcript in normalized SAGE libraries is a measure of a change in its abundance as a proportion of the transcriptome, and therefore these numbers do not suggest that transcription is generally suppressed by antibody treatment. In fact, activated cells generally appear to transcribe many more RNA molecules than resting cells. For example, in this study, 2.84 and $6.7 \mu \mathrm{g}$ of total RNA were extracted per million cells from resting and activated clone 32 cells, respectively.

A measure of the similarity in the overall scale of the changes for a given pair of libraries is also given by simple Pearson correlation coefficients. These correlation coefficients are $R=0.68$ for the comparison of the libraries derived from resting and anti-CD8 antibody-treated clone $32 \mathrm{~T}-$ cells, and $R=0.56$ for comparisons of the resting and antiCD3 antibody-treated libraries. This suggests that, although similar numbers of tags are significantly affected in each case, the transcriptional response of the cells to anti-CD8 antibodies is somewhat weaker than its response to antiCD3 antibodies. The correlations for the resting clone 32 library versus libraries derived from other cell types show larger overall differences: $R \sim 0.5$ for the comparisons with a CD $4^{+}$T-cell-derived library and $R \sim 0.4$ for the comparison with a cerebellum-derived library [9]. This contrasts with the similarities of, e.g., the library derived from clone 32 cells versus another derived from natural killer cells, which differ by less than 200 significantly over-represented tags and for which the overall tag distributions are highly correlated $(R \sim 0.8)$, once again emphasizing the similarities between these two lineages [9].

These results indicate that anti-CD8 antibody treatment induces considerable transcriptional remodeling in clone $32 \mathrm{~T}$-cells, without substantially altering the expression of most of the surface markers tested by FACS (Supplementary information, Figure S1). The main exception is CD8, which is dramatically down-regulated at the cell surface. This is likely to be due to receptor internalization following antibody ligation. Smaller decreases in the abundance of surface CD3 and CD5 are also observed. This may be due to the weak direct or indirect association of these molecules with CD8 [11], as it is internalized, or it could represent 'real' changes induced by signaling. We analyzed the SAGE data for evidence of transcriptional regulation of the genes encoding all the surface antigens tested by FACS (Supplementary information, Figure S2). Unsurprisingly, given the well-documented, poor correlation between mRNA and protein expression levels [12], there are changes in the transcription of some of these genes following anti-CD8 antibody treatment. In most cases, however, a $\leq 2$-fold decrease in transcript level correlated well with very small reductions in surface expression, suggesting that mRNA levels have to decrease more substantially in order to become limiting. The transcription of four genes does not correlate well with the FACS data: CD3 $\varepsilon$ transcripts fall by $\sim 5$-fold on antibody treatment, whereas surface CD3 levels fall only marginally (and this may only be due to protein internalization as discussed above); CD8 $\alpha$ transcripts increase by $\sim 5$-fold despite the large fall in surface expression level; and CD45 and CD122 transcript numbers increase by $\sim 4-$ and $\sim 5$ fold, respectively, without their protein expression levels 
changing. The discrepancy in CD8 levels is, as discussed, explained by receptor internalization following ligation by antibody, which may override increases in transcription. The surface expression of the CD3/TCR complex is known to depend on the presence of all components; only CD3 $\varepsilon$ transcription decreases substantially in this instance, which might not be expression limiting. CD122 is also found in heterodimeric complexes with CD132 at the cell surface; so its levels may be limited by CD132 transcription. It is unclear why no change in CD45 surface expression is observed. Given the well-described, very high levels of CD45 protein expression [13], a further large increase in the expression of this protein at the cell surface may not be possible. Significantly, transcription of the genes encoding the activation markers CD25 and CD69 is not enhanced by anti-CD8 antibody treatment, in marked contrast to the effects of anti-CD3 antibody treatment (Supplementary information, Figure S2).

We also addressed whether or not, alongside the comparable quantitative gene expression changes that we have observed, there are qualitatively similar transcriptional changes, i.e. whether the same types of transcripts are upor down-regulated, in response to anti-CD8 and anti-CD3 antibody treatment. Pearson correlation analysis of the antiCD8 and anti-CD3 antibody-treated clone $32 \mathrm{~T}$-cell-derived libraries revealed that, in terms of the scale of the global differences, the anti-CD8 and anti-CD3 antibody-treated cells are more similar to each other $(R=0.72)$ than they are to the resting cells ( $R=0.68$ and 0.56 , respectively). Nonetheless, there are large differences in the transcriptional responses of clone 32 to the two antibody treatments.
Approximately 450 tags are significantly more abundant following anti-CD8 antibody treatment than after anti-CD3 antibody treatment, and more than 500 are significantly less abundant (Figure 1C). Moreover, of the 1766 tags whose abundance is significantly increased or decreased in either the anti-CD8 or the anti-CD3 antibody-treated clone 32 T-cell-derived SAGE libraries versus the resting clone 32 T-cell-derived library, only one-third (i.e. 601) are shared. Of these shared tags, more than two-thirds (i.e. 430) are significantly less well represented in each library compared to the resting library at the $1 \%$ confidence level rather than over represented, suggesting that there is greater similarity between the antibody treatments in terms of their suppression of gene expression rather than its induction.

Inspection of the tags in the SAGE libraries whose representation was significantly altered ( $p$-value $\leq 0.01$ according to the Audic-Claverie (AC) test) by one or the other of the antibody treatments (Figure 2A) further emphasizes the finding that relatively few tags change abundance to similar extents in both libraries (such genes have the same color-coding in Figure 2A). Nevertheless, for most tags that vary in abundance, it is only the degree of over- or under-representation that changes: versus the resting clone 32 T-cell-derived library, relatively few tags are over-represented following treatment with one antibody and under-represented following treatment with the other (e.g., change from being yellow-coded to being blue-coded in Figure 2A, or vice versa). The only obvious exception is a set of tags (blue bars in the bracketed region of Figure 2A) that are unchanged or over-represented after antiCD3 treatment and under-represented following anti-CD8
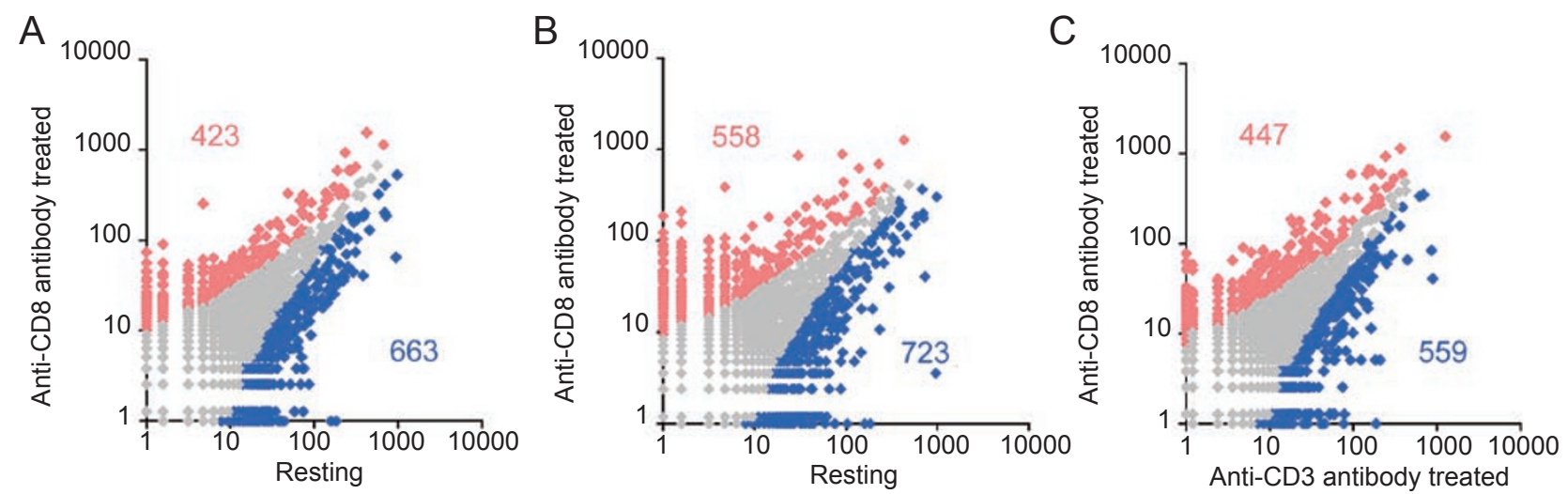

Figure 1 Comparisons of resting and antibody-treated clone 32 SAGE libraries. For each 10-bp SAGE tag, its abundance per 100000 tags in the libraries derived from anti-CD8 antibody-treated versus resting clone 32 cells (A), those derived from anti-CD3 antibody-treated versus resting clone 32 cells $(B)$ or those derived from anti-CD8 antibody-treated versus anti-CD3 antibody-treated clone 32 cells (C) is plotted on a logarithmic scale. Zero values are plotted as 1 tag per 100000 . The symbols (diamonds) representing each tag are coloured according to the statistical significance of the apparent differential abundance of the tag, calculated using the method of Audic and Claverie [11]. Red and blue symbols represent tags that are significantly more abundant in one of the two libraries being compared with $p$-values of $<1 \%$, with the number of tags in each group given alongside. 
A Anti-CD3 anti-CD8

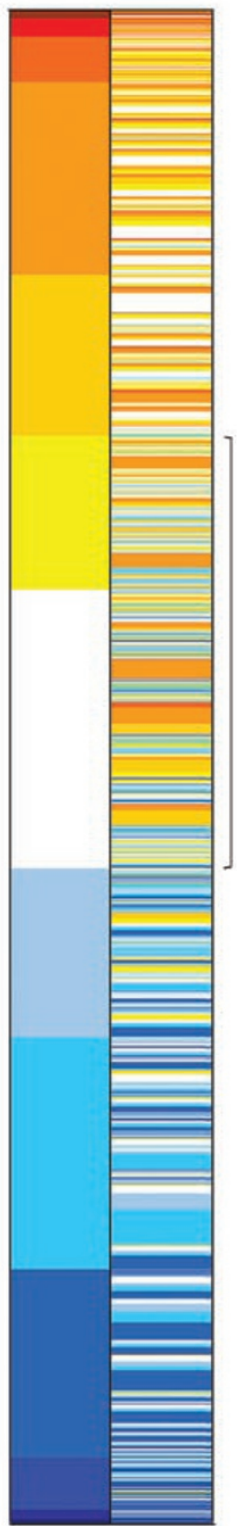

B

Anti-CD3 anti-CD8

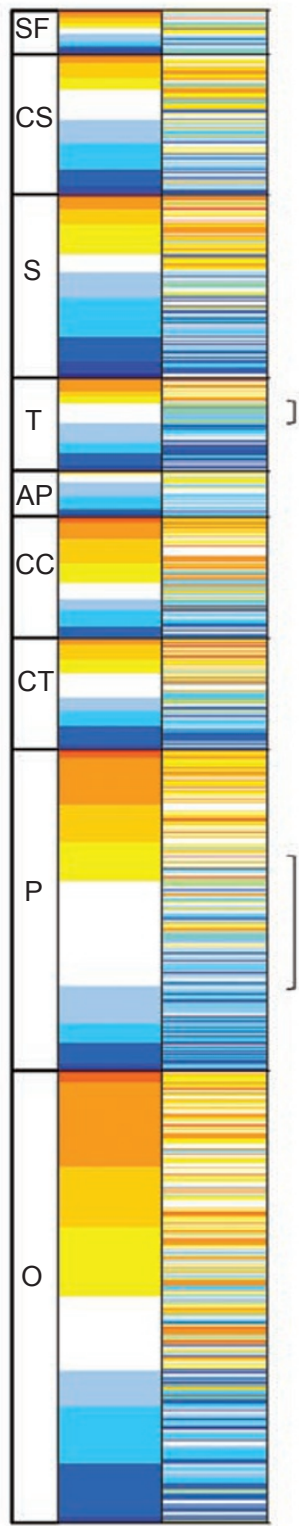

C

Anti-CD3 anti-CD8
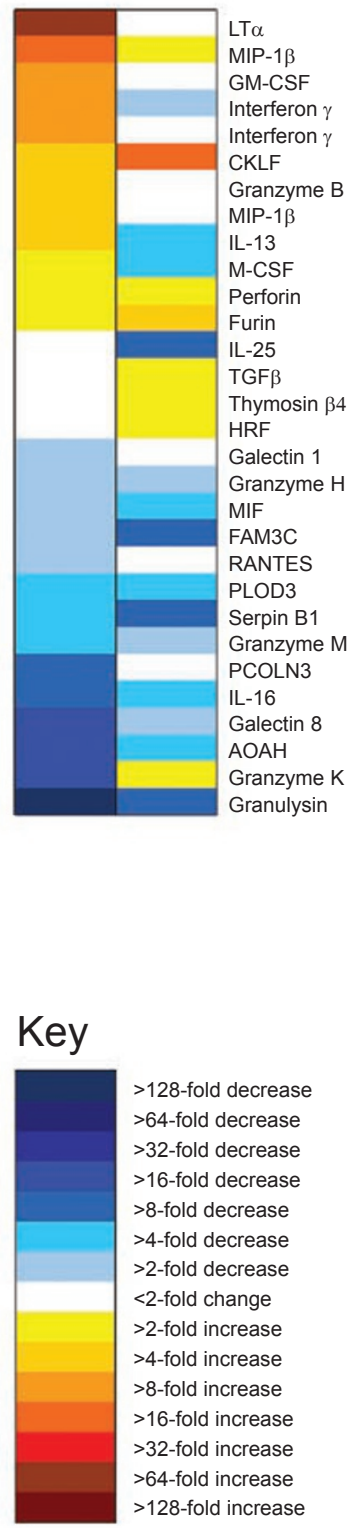

D

Anti-CD3 anti-CD8

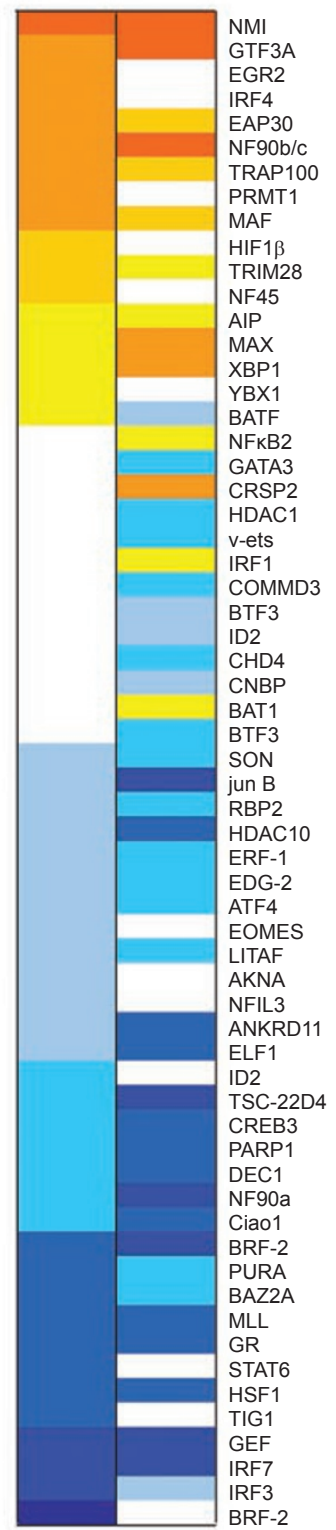

Figure 2 Comparison of transcripts whose expression in clone 32 is affected by anti-CD8 and anti-CD3 antibody treatments. Each line in the plots represents a unique SAGE tag sequence, colored according to the fold change in its relative abundance in the antibody-treated library indicated at the top of the column compared to that in the resting clone 32-derived library. Coloring is on a log base 2 scale, as shown in the key below panel C. Bracketed regions are referred to in the text. In (A), all 2472 tags that are significantly differentially abundant in either comparison are listed, and sorted according to the fold change in abundance in the anti-CD3 antibody-treated library compared to the resting cell-derived library. In (B), only the subset of those tags from (A) that have been assigned to a transcript encoding a protein of known function are included and they are sorted first by the functional class of this protein, then by the fold change in abundance in the library derived from anti-CD3 antibody-treated clone 32 cells versus those derived from resting clone 32 cells, as in (A). Codes used for the functional classes are as follows: SF, secreted factors; CS, cell surface molecules; S, cytoplasmic signaling molecules; T, transcriptional regulation; AP, antigen presentation; CC, cell cycle and survival; CT, cytoskeleton and vesicle transport; $\mathrm{P}$, protein synthesis; and $\mathrm{O}$, other function, including many metabolic enzymes and other housekeeping genes. (C and D) identify all the tags from (B) linked to secreted factors and proteins involved in transcriptional regulation, respectively. In these panels, the common abbreviation for the protein encoded by the transcript linked to the tag is given alongside. If a protein name appears twice, two SAGE tags associated with its transcript were significantly differentially expressed in one of the comparisons. The numerical values of the changes in abundance for all the SAGE tags used in this figure, together with their sequence and the description of all UniGene clusters automatically linked to them, are available in Supplementary information, Spreadsheet 1. 
treatment, thus revealing a somewhat suppressive effect of anti-CD8 antibody treatment. When the tags are ordered according to the putative functions of their associated transcripts (Figure 2B-2D), it is clear that there are differences in the representation of tags corresponding to transcripts with obvious T-cell-associated functions, e.g. cell surface molecules, signaling molecules and secreted factors (Figure 2B-2C; Supplementary information, Figure S3 and Spreadsheet 1), as well as those linked with more generic functions, e.g. the category of "other known functions" (mainly housekeeping genes; Figure 2B; Supplementary information, Spreadsheet 1).

Consideration of the tags whose abundances change significantly and that correspond to genes of known function (Figure 2B-2D) yields three striking observations. First, there is very little induction of the expression of soluble effector molecules by anti-CD 8 antibody treatment (Figure $2 \mathrm{C}$ ): only 4 of the 12 transcripts that are $>2$-fold up-regulated by anti-CD3 treatment are affected at all by anti-CD 8 antibody treatment. Second, a tag associated with TGF $\beta$, which is generally linked with immunosuppressive effects [14], is $>3$-fold over-represented in the library derived from anti-CD8 antibody-treated cells but unchanged in the library produced from anti-CD3 antibody-treated cells (Figure 2C). Third, a large number of tags associated with transcription and protein synthesis (bracketed regions in Figure 2B, but also see Figure 2D) have reduced abundance in the library derived from anti-CD8 antibody-treated cells but are not similarly affected by anti-CD3 treatment. Indeed, $69 \%$ and $55 \%$, respectively, of all the tags that are associated with protein synthesis or transcription factors and exhibit either $\mathrm{a} \leq 2$-fold change in abundance or an increase in abundance following anti-CD3 antibody treatment versus resting clone 32 cells are reduced in abundance in the library derived from anti-CD8 antibody-treated cells. This compares to an average of only $27 \%$ for all the other categories. One explanation for this is that the anti-CD8 antibody induced the expression of factors that inhibit T-cell activation and, in particular, proliferation, which would otherwise require an increase in the rate of protein synthesis. In this context, it is interesting that tags corresponding to two molecules with well-defined roles in inhibiting T-cell activation, i.e. diacylglycerol kinase $\zeta[15]$ and casein kinase $1 \alpha$ [16], exhibit greater abundance in the library derived from anti-CD 8 antibody-treated clone 32 cells versus the library produced from resting cells (an $\sim 11$-fold increase and a change from 0 to 7.7 tags per 100000 , respectively; Figure $3)$. More modest changes accompanied anti-CD3 antibody treatment (an $\sim 3$-fold increase and a change from 0 to 4.8 tags per 100 000, respectively; Figure 3).

Having shown that anti-CD8 antibody treatment induces substantial changes in gene transcription, the question arises as to how CD8 generates signals capable of achieving this. Our findings allow for either of two interesting possibilities. The first possibility is that CD8 may be capable of signaling entirely independently of the TCR. This seems rather unlikely, however, given that the signals induced by CD8 ligation are somewhat inhibitory, whereas it is known that, by binding to the same MHC protein, CD8 greatly enhances coincident activating signaling by the

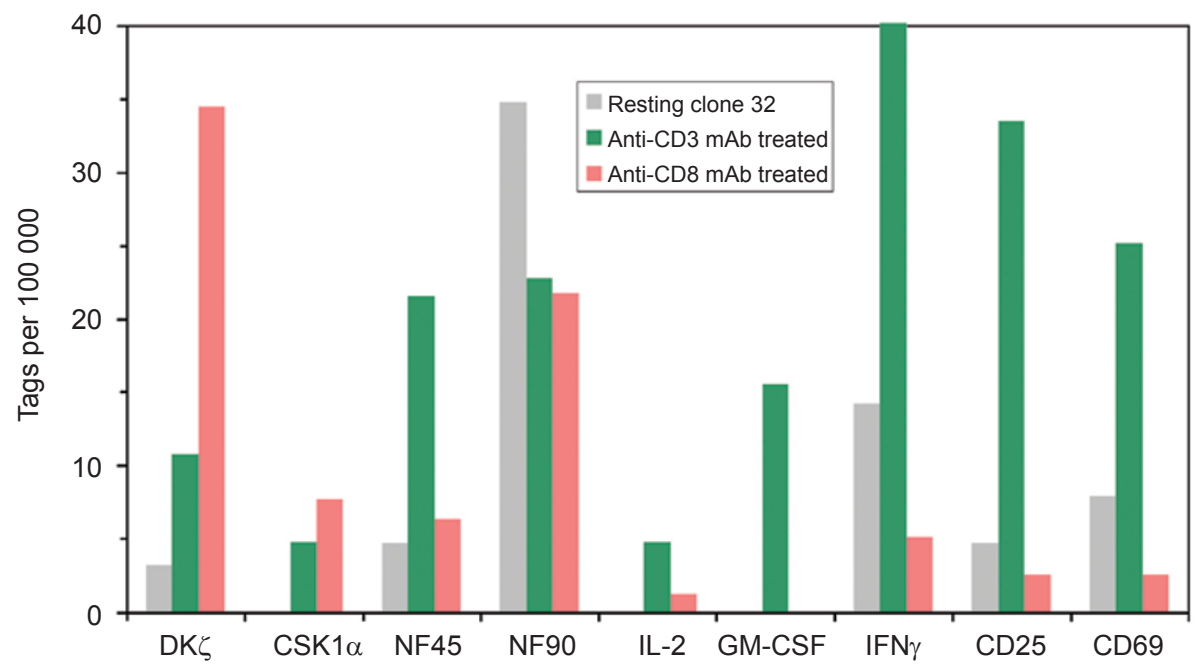

Figure 3 Histogram showing total tag abundance for transcripts of interest in resting and antibody-treated clone 32-derived SAGE libraries. Normalized tag counts (i.e. tag numbers expressed as tags per 100000 ) in the SAGE libraries derived from resting, anti-CD8 antibody-treated and anti-CD3 antibody-treated clone 32 cells, for all SAGE tags linked to all the known transcripts associated with the genes indicated, were summed and plotted on a linear scale. The total count for IFNy associated tags in the anti-CD3 antibody-treated library was 196 tags per 100000. 
TCR (reviewed in [17]).

The second possibility is that CD8 signals indirectly via the TCR. It has been proposed that CD8 associates, at least weakly, with the TCR since CD3/TCR is co-immunoprecipitated with anti-CD8 antibodies [18]. How would this induce signaling? In situations of this type, anti-CD8 antibodies would be expected to cross-link TCRs, increasing their local concentration versus antagonistic phosphatases, such as CD45, giving net signaling locally [19]. Since only a fraction of the TCRs are associated with CD8, it could be expected that the transcriptional changes thus induced, although weaker, would mirror those induced via the TCR directly. On the other hand, comparison of signaling by TCR agonists and antagonists suggests that signals of different strength transmitted via the same TCR can induce profoundly different outcomes $[20,21]$. What we observe following anti-CD8 antibody treatment is transcriptional remodeling at the scale observed in response to signaling through the TCR, but without overt activation, a situation that parallels antagonist signaling in some respects. The induction of inhibitory factors such as diacylglycerol kinase $\zeta$ and casein kinase $1 \alpha$, and the suppression of a subset of transcripts associated with protein synthesis, each suggest that a distinct inhibitory program is invoked by CD8 ligation.

Several clues in our data suggest that the failure to induce formation of an active NFAT transcription complex is at least part of the explanation for why CD8 ligation fails to activate the T-cell clone. First, casein kinase $1 \alpha$, which phosphorylates NFAT4 and prevents its nuclear translocation [16], appears to be upregulated to a greater extent by anti-CD 8 treatment than by conventional activation. Second, whereas the abundance of the tag corresponding to the transcript encoding NF45 increased by $\sim 5$-fold following anti-CD3 treatment, it is unchanged by anti-CD8 antibody treatment (Figures 2D and 3). NF45 (also known as ILF2) is a $45 \mathrm{kDa}$ subunit of the NFAT complex known to be required for the induction of, among others, IL-2 gene transcription [22]. The various splice forms of NF90 (ILF3), which pairs with NF45 in the complex, are similarly regulated by both antibody treatments (Figures 2D and 3), but the implications of this are unclear given that the activity of this constitutively expressed subunit is not thought to be regulated at the level of its own transcription but rather via its association with NF45 [23] and by nuclear export [24]. Finally, tags associated with several NFATregulated genes that are over-represented in the anti-CD3 antibody-treated library versus the resting library are either unaffected or have reduced abundance following anti-CD8 antibody treatment (Figure 3). These include IL-2 (absent in the resting library and present at 1 and 5 tags per 100000 in the anti-CD8 and anti-CD3 antibody-treated libraries, respectively), GM-CSF (absent in the resting and antiCD8 antibody-treated libraries and present at 15 tags per 100000 in the anti-CD3 antibody-treated library), IFN- $\gamma$ (increased by 13 -fold following anti-CD3 antibody treatment and decreased by 3 -fold following anti-CD8 antibody treatment), CD25 (IL-2R $\alpha$; increased by 7-fold following anti-CD3 antibody treatment and decreased by 2 -fold after anti-CD8 antibody treatment) and CD69 (increased by 3fold following anti-CD3 antibody treatment and decreased by 3 -fold by anti-CD 8 antibody treatment). We speculate that ligating CD8 alone induces weak signals with an inhibitory character reminiscent of the effects of antagonists $[20,21,25]$, although the detailed nature of these signals must await further investigation.

Final question prompted by the unexpected breadth of the expression changes we see is to what extent are these changes part of a programmed signaling response or the result of stochastic effects on transcription? Recent studies of transcriptional remodeling in response to environmental stress suggest that it involves non-specific, global gene expression changes that affect a large fraction of the transcriptome and are stochastic in nature, i.e. they are not reproducible even when experimental conditions do not vary $[26,27]$. In one study, only $10 \%$ of the changes observed were consistently reproducible [27]. However, this is not always the case [28], and the ratio of 'neutral' (stochastic) versus 'adaptive' (reproducible and functionally relevant) changes may depend on (1) the strength of the perturbation or (2) whether or not the cell has adapted to making that response through evolution, or both (discussed in [29]). It would be of great interest to determine whether similar effects accompany receptor signaling, thus explaining the large number of transcriptional changes we observe. It is not feasible to address this using SAGE, but microarray assays using multiple replicates could be expected to distinguish between adaptive and stochastic responses, by identifying reproducible changes.

In conclusion, our results highlight three aspects of anti-CD8 antibody-induced signaling in the $\mathrm{CD} 8^{+} \mathrm{T}$-cell clone we have studied. First, even though it fails to induce detectable phenotypic changes, for example, by FACS analysis of cell surface markers, CD8 ligation with antibodies profoundly alters transcription. Second, the scale of the transcriptional remodeling is comparable to that induced by anti-CD3 antibody treatment. Third, the character of the resulting changes is distinct, with our preliminary analysis suggesting that the effects of the anti-CD8 antibody are rather more inhibitory than those resulting from anti-CD3 antibody-induced signaling. The degree to which stochastic effects may exaggerate these differences is unclear, but $\mathrm{CD} 8$ is clearly very different from $\mathrm{CD} 28$, which is largely incapable of independent signaling in response to 
reagents, such as non-superagonistic antibodies, used to mimic native ligands $[30,31]$. When CD28 is induced to signal, i.e. by cross-linking it along with CD3, the large set of genes whose expression changes dramatically is essentially identical to that induced, albeit much more weakly, by anti-CD3 antibody treatment alone. Further work with other T-cell populations and alternative means of stimulating responses via CD8, with and without concomitant TCR ligation, will likely provide new insights into the mechanism and possible physiological significance of CD8-mediated signaling.

\section{Materials and Methods}

\section{$C D 8^{+}$T-cell clone 32 and SAGE library construction}

All SAGE libraries were produced from RNA extracted from clone 32, a cytotoxic T-cell clone specific for the HIV-1 Pol peptide ETAYFILKL presented in the context of HLA A68. Clone 32 production [8] and generation of the SAGE library from resting clone 32 cells [9] have been described previously. The anti-CD8 antibody-treated clone 32 SAGE library was produced according to the standard protocol using the I-SAGE kit (Invitrogen, Paisley, UK) from RNA extracted from clone 32 cells treated with $5 \mu \mathrm{g} / \mathrm{ml}$ of MF8 anti-CD8 antibody (obtained in the form of ascites from the Third Leukocyte Typing Workshop [32]) in solution for $48 \mathrm{~h}$. The standard SAGE tag library derived from anti-CD3 antibody-treated cells was computationally generated from a LongSAGE library produced according to the protocol provided with the LongSAGE kit (Invitrogen) from $10.9 \mu \mathrm{g}$ of total RNA extracted from clone 32 cells treated with $30 \mathrm{ng} / \mathrm{ml}$ of OKT3 anti-CD3 antibody (eBiosciences, San Diego, CA) attached to Dynalbeads (Invitrogen), for 24 h. The final SAGE libraries consisted of 63270,78290 and 83556 conventional (10 base) SAGE tags for the resting, MF8 antibodytreated and OKT3 antibody-treated clone 32 cells, respectively. The high purity of the cells used for production of the resting clone 32 library has been demonstrated [9] and no additional cells were added in the course of either antibody treatment.

\section{Analysis of SAGE sequences}

The SAGE 2000 program (obtained from Dr K Kinzler, Johns Hopkins University) was used to extract ditags from the concatamer sequences, exclude duplicate ditags (which are likely to have arisen from PCR bias) and produce a list of unique SAGE tag sequences together with their frequency of observation, referred to here as the "tag count". Inter-library comparisons were performed as previously described [9]. The Audic-Claverie (AC) test [33] was used for assessing the significance of observed differences in tag abundance between pairs of libraries. However, due to software limitations, the AC test could not be used for tags occurring more than 140 times in the two libraries. For these tags the $\chi^{2} 2 \times 2$ test is expected to be most accurate [34] and was used. SAGE tags of interest (i.e. all tags whose abundance was significantly different between two libraries at the $1 \%$ level) were linked to the SAGEmap Tag to UniGene Mapper [35] and SAGE Genie [36] to identify the potential transcripts from which the tags are likely to have derived. The transcript matches were then manually curated as follows: apparent Unigene [37] matches were checked to ensure that the tag did not match only a single EST, only $5^{\prime}$ ESTs (i.e. the tag was not truly at the $3^{\prime}$-most NlaIII site),
ESTs which had been incorrectly clustered or those that appeared to contain single-base errors compared to the "true transcript" (i.e. the sequence represented by the mRNAs and the majority of ESTs in the cluster). Once transcripts had been confidently matched to tags, their functions were categorized based on their Unigene descriptions, Locuslink and RefSeq [38] entries where available and, where necessary, information from the literature.

\section{Statistical comparisons of SAGE libraries}

The SAGE library from activated $\mathrm{CD}^{+} \mathrm{T}$-cells was obtained from the University of Tokyo (www.prevent.m.u-tokyo.ac.jp/sage. html) and the library from cerebellum was obtained from CGAP [39] via SAGEmap [35]. Pearson correlation coefficients, which were used for overall comparisons of all the normalized tag abundances between libraries, were calculated using SPSS 11.0 (SPSS Inc., Chicago, IL).

\section{References}

1 Holler PD, Kranz DM. Quantitative analysis of the contribution of TCR/pepMHC affinity and CD8 to T cell activation. Immunity 2003; 18:255-264.

2 Wyer JR, Willcox BE, Gao GF, et al. T cell receptor and coreceptor CD8 alphaalpha bind peptide-MHC independently and with distinct kinetics. Immunity 1999; 10:219-225.

3 Davis SJ, Ikemizu S, Evans EJ, Fugger L, Bakker TR, van der Merwe PA. The nature of molecular recognition by T cells. Nat Immunol 2003; 4:217-224.

4 Leishman AJ, Naidenko OV, Attinger A, et al. T cell responses modulated through interaction between CD8alphaalpha and the nonclassical MHC class I molecule, TL. Science 2001; 294:19361939.

5 Tsujimura K, Obata Y, Matsudaira Y, et al. The binding of thymus leukemia (TL) antigen tetramers to normal intestinal intraepithelial lymphocytes and thymocytes. J Immunol 2001; 167:759-764.

6 Spruyt LL, Glennie MJ, Beyers AD, Williams AF. Signal transduction by the $\mathrm{CD} 2$ antigen in $\mathrm{T}$ cells and natural killer cells: requirement for expression of a functional $\mathrm{T}$ cell receptor or binding of antibody $\mathrm{Fc}$ to the Fc receptor, Fc gamma RIIIA (CD16). J Exp Med 1991; 174:1407-1415.

7 Luhder F, Huang Y, Dennehy KM, et al. Topological requirements and signaling properties of T cell-activating, anti-CD28 antibody superagonists. J Exp Med 2003; 197:955-966.

8 Moss PA, Rowland-Jones SL, Frodsham PM, et al. Persistent high frequency of human immunodeficiency virus-specific cytotoxic T cells in peripheral blood of infected donors. Proc Natl Acad Sci USA 1995; 92:5773-5777.

9 Evans EJ, Hene L, Sparks LM, et al. The T cell surface - how well do we know it? Immunity 2003; 19:213-223.

10 Velculescu VE, Zhang L, Vogelstein B, Kinzler KW. Serial analysis of gene expression. Science 1995; 270:484-487.

11 Beyers AD, Spruyt LL, Williams AF. Molecular associations between the T-lymphocyte antigen receptor complex and the surface antigens CD2, CD4, or CD8 and CD5. Proc Natl Acad Sci USA 1992; 89:2945-2949.

12 Gygi SP, Rochon Y, Franza BR, Aebersold R. Correlation between protein and mRNA abundance in yeast. Mol Cell Biol 1999; 19:1720-1730. 
13 Williams AF, Barclay AN. Glycoprotein antigens of the lymphocyte surface and their purification by antibody affinity chromatography. In: Weir DM, Herzenberg LA, eds. Handbook of experimental immunology. Oxford: Blackwell Scientific Publishing, 1985: 22.1-22.24.

14 Wahl SM, Wen J, Moutsopoulos N. TGF-beta: a mobile purveyor of immune privilege. Immunol Rev 2006; 213:213-227.

15 Zhong XP, Hainey EA, Olenchock BA, et al. Enhanced T cell responses due to diacylglycerol kinase zeta deficiency. Nat Immunol 2003; 4:882-890.

16 Zhu J, Shibasaki F, Price R, et al. Intramolecular masking of nuclear import signal on NF-AT4 by casein kinase I and MEKK1. Cell 1998; 93:851-61.

17 Gao GF, Rao Z, Bell JI. Molecular coordination of alphabeta Tcell receptors and coreceptors CD8 and CD4 in their recognition of peptide-MHC ligands. Trends Immunol 2002; 23:408-413.

18 Doucey MA, Goffin L, Naeher D, et al. CD3 delta establishes a functional link between the T cell receptor and CD8. J Biol Chem 2003; 278:3257-3264.

19 Davis SJ, van der Merwe PA. The kinetic-segregation model: TCR triggering and beyond. Nat Immunol 2006; 7:803-809.

20 Klenerman P, Rowland-Jones S, McAdam S, et al. Cytotoxic T-cell activity antagonized by naturally occurring HIV-1 Gag variants. Nature 1994; 369:403-407.

21 Sykulev Y, Vugmeyster Y, Brunmark A, Ploegh HL, Eisen HN. Peptide antagonism and T cell receptor interactions with peptideMHC complexes. Immunity 1998; 9:475-483.

22 Zhao G, Shi L, Qiu D, Hu H, Kao PN. NF45/ILF2 tissue expression, promoter analysis, and interleukin-2 transactivating function. Exp Cell Res 2005; 305:312-323.

23 Reichman TW, Muniz LC, Mathews MB. The RNA binding protein nuclear factor 90 functions as both a positive and negative regulator of gene expression in mammalian cells. Mol Cell Biol 2002; 22:343-356.

24 Shim J, Lim HR, Yates J, Karin M. Nuclear export of NF90 is required for interleukin-2 mRNA stabilization. Mol Cell 2002; 10:1331-1344.

25 Dittel BN, Stefanova I, Germain RN, Janeway CA Jr. Cross-antagonism of a $\mathrm{T}$ cell clone expressing two distinct $\mathrm{T}$ cell receptors. Immunity 1999; 11:289-298.

26 Fong SS, Joyce AR, Palsson BO. Parallel adaptive evolution cultures of Escherichia coli lead to convergent growth phenotypes with different gene expression states. Genome Res 2005; 15:1365-1372.

27 Stern S, Dror T, Stolovicki E, Brenner N, Braun E. Genome-wide transcriptional plasticity underlies cellular adaptation to novel challenge. Mol Syst Biol 2007; 3:106.

28 Pelosi L, Kuhn L, Guetta D, et al. Parallel changes in global protein profiles during long-term experimental evolution in Escherichia coli. Genetics 2006; 173:1851-1869.

29 Koonin EV. Chance and necessity in cellular response to challenge. Mol Syst Biol 2007; 3:107.

30 Riley JL, Mao M, Kobayashi S, et al. Modulation of TCR-induced transcriptional profiles by ligation of CD28, ICOS, and CTLA-4 receptors. Proc Natl Acad Sci USA 2002; 99:11790-11795.

31 Diehn M, Alizadeh AA, Rando OJ, et al. Genomic expression programs and the integration of the CD28 costimulatory signal in T cell activation. Proc Natl Acad Sci USA 2002; 99:1179611801.

32 McMichael AJ, Beverley PCL, Cobbold S, et al., eds. Leucocyte typing III: white cell differentiation antigens. Oxford: Oxford University Press, 1987.

33 Audic S, Claverie JM. The significance of digital gene expression profiles. Genome Res 1997; 7:986-995.

34 Romualdi C, Bortoluzzi S, Danieli GA. Detecting differentially expressed genes in multiple tag sampling experiments: comparative evaluation of statistical tests. Hum Mol Genet 2001; 10:2133-2141.

35 Lash AE, Tolstoshev CM, Wagner L, et al. SAGEmap: a public gene expression resource. Genome Res 2000; 10:1051-1060.

36 Boon K, Osorio EC, Greenhut SF, et al. An anatomy of normal and malignant gene expression. Proc Natl Acad Sci USA 2002; 99:11287-11292.

37 Wheeler DL, Barrett T, Benson DA, et al. Database resources of the National Center for Biotechnology Information. Nucleic Acids Res 2006; 34:D173-D180.

38 Pruitt KD, Maglott DR. RefSeq and LocusLink: NCBI genecentered resources. Nucleic Acids Res 2001; 29:137-140.

39 Riggins GJ, Strausberg RL. Genome and genetic resources from the Cancer Genome Anatomy Project. Hum Mol Genet 2001; 10:663-667.

(Supplementary Information is linked to the online version of the paper on the Cell Research website.) 